\title{
Association between Inpatient Delirium and Hospital Readmission in Patients $\geq 65$ Years of Age: A Retrospective Cohort Study
}

\author{
Sara C LaHue, MD ${ }^{1,2}$; Vanja C. Douglas, MD,2; Teresa Kuo, MD³; Carol A Conell, PhD; Vincent X Liu, MD, MS4; \\ $\mathrm{S}$ Andrew Josephson, MD ${ }^{1,2}$; Clay Angel, MD; Kristen B Brooks, MD
}

${ }^{1}$ Department of Neurology, School of Medicine, University of California, San Francisco, California; ${ }^{2}$ Weill Institute for Neurosciences, Department of Neurology, University of California, San Francisco, California; ${ }^{3}$ Department of Medicine, Kaiser Permanente San Francisco Medical Center, San Francisco, California; ${ }^{4}$ Division of Research, Kaiser Permanente Northern California, Oakland, California; ${ }^{5}$ Department of Hospital Medicine, Kaiser Permanente San Rafael Medical Center, San Rafael, California; ${ }^{6}$ Department of Psychiatry, Kaiser Permanente San Rafael Medical Center, San Rafael, California.

BACKGROUND: Delirium affects more than seven million hospitalized adults in the United States annually. However its impact on postdischarge healthcare utilization remains unclear.

OBJECTIVE: To determine the association between delirium and 30-day hospital readmission.

DESIGN: A retrospective cohort study.

SETTING: A general community medical and surgical hospital.

PATIENTS: All adults who were at least 65 years old, without a history of delirium or alcohol-related delirium, and were hospitalized from September 2010 to March 2015.

MEASUREMENTS: The patients deemed at risk for or displaying symptoms of delirium were screened by nurses using the Confusion Assessment Method with a followup by a staff psychiatrist for a subset of screen-positive patients. Patients with delirium confirmed by a staff psychiatrist were compared with those without delirium.
The primary outcome was the 30-day readmission rate. The secondary outcomes included emergency department (ED) visits 30 days postdischarge, mortality during hospitalization and 30 days postdischarge, and discharge location.

RESULTS: The cohort included 718 delirious patients and 7,927 nondelirious patients. Using an unweighted multivariable logistic regression, delirium was determined to be significantly associated with the increased odds of readmission within 30 days of discharge (odds ratio (OR): 2.60; 95\% Cl, 1.96-3.44; $P<.0001)$. Delirium was also significantly $(P<.0001)$ associated with ED visits within 30 days postdischarge (OR: $2.18 ; 95 \% \mathrm{Cl}: 1.77-2.69$ ) and discharge to a facility (OR: 2.52; 95\% Cl: 2.09-3.01).

CONCLUSIONS: Delirium is a significant predictor of hospital readmission, ED visits, and discharge to a location other than home. Delirious patients should be targeted to reduce postdischarge healthcare utilization. Journal of Hospital Medicine 2019;14:201-206. (C) 2019 Society of Hospital Medicine

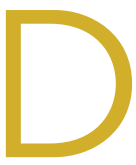

elirium is an acute change in mental status, affecting more than seven million hospitalized patients in the United States annually. ${ }^{1}$ Several factors increase the risk of developing delirium, including advanced age, ${ }^{2}$ cognitive dysfunction, ${ }^{3}$ hearing and vision impairment, ${ }^{4-6}$ and severe illness or major surgery. ${ }^{7}$ Delirium may be precipitated during hospitalization by common inpatient interventions, such as the use of physical restraints, polypharmacy, or bladder catheters. $^{4,8}$ In-hospital delirium impacts an estimated 10\%-15\% of the general medical admissions and as many as $81 \%$ of patients in the intensive care unit (ICU). ${ }^{9-11}$ Despite the relative frequency

\footnotetext{
*Corresponding Author: Sara Catherine LaHue, MD; E-mail: Sara.LaHue@ucsf edu; Telephone: 415-476-1489.

Additional Supporting Information may be found in the online version of this article.
}

Received: June 17, 2018; Revised: October 26, 2018;

Accepted: November 20, 2018

(c) 2019 Society of Hospital Medicine DOI 10.12788/jhm.3130 with which delirium is encountered in the hospital, subsequent emergency department (ED) presentations or hospital readmissions for these patients are poorly characterized.

The development of delirium is associated with several negative outcomes during the hospital stay. Delirium is an independent predictor of prolonged hospital stay, ${ }^{7,9,12,13}$ prolonged mechanical ventilation, ${ }^{14}$ and mortality during admission. ${ }^{14,15}$ Inpatient delirium is associated with functional decline at discharge, leading to a new nursing home placement. ${ }^{16-19}$ Preexisting dementia is exacerbated by inpatient delirium, and a new diagnosis of cognitive impairment ${ }^{20}$ or dementia becomes more common after an episode of delirium. ${ }^{21}$

These data suggest that people diagnosed with delirium may be particularly vulnerable in the posthospitalization period. Hospitals with high rates of unplanned readmissions face penalties from the Centers for Medicare and Medicaid Services. ${ }^{22,23}$ However, few investigations have focused on postdischarge healthcare utilization, such as readmission rates and ED visits. Studies that address this topic are limited to postoperative patient populations. ${ }^{24}$ 


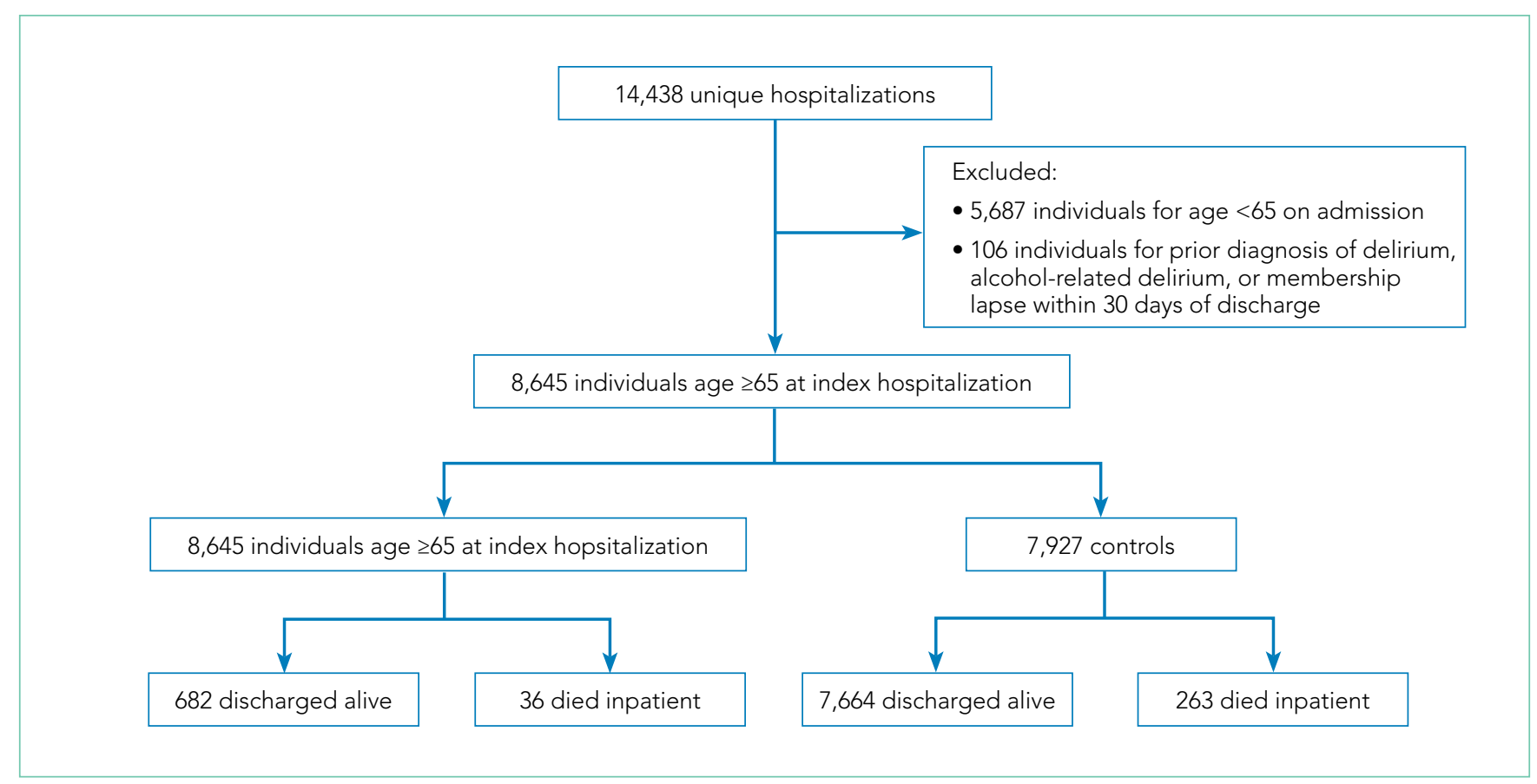

FIG. Subject acquisition flow diagram.

Using a cohort of hospitalized patients, we examined whether those diagnosed with delirium experienced worse outcomes compared with patients with no such condition. We hypothesized that the patients diagnosed with delirium during hospitalization would experience more readmissions and ED visits within 30 days of discharge compared with those without delirium.

\section{METHODS}

\section{Study Design}

This single-center retrospective cohort study took place at the Kaiser Permanente San Rafael Medical Center (KP-SRF), a 116bed general community medical and surgical hospital located in Northern California, from September 6, 2010 to March 31, 2015. The Kaiser Permanente Northern California institutional review board, in accordance with the provisions of the Declaration of the Helsinki and International Conference on Harmonization Guidelines for Good Clinical Practice (CN-152491-H), approved this study.

\section{Participants and Eligibility Criteria}

This study included Kaiser Permanente members at least 65 years old who were hospitalized at KP-SRF from September 2010 to March 2015. Patient data were obtained from the electronic medical records. Patients with delirium were identified from a delirium registry; all other patients served as controls.

Starting on September 6, 2010, a hospital-wide program was initiated to screen hospitalized medical and surgical patients using the Confusion Assessment Method (CAM). ${ }^{25}$ As part of this program, nurses completed a four-hour training on delirium; the program included delirium identification and CAM administration. Patients deemed at risk for delirium by their nurse or displaying symptoms of delirium (fluctuation in attention or awareness, disorientation, restlessness, agitation, and psychomotor slowing) were screened by nurses one to two times within a 24-hour period. Physicians were notified by the nurse if their patient screened positive. Nurses were prohibited from performing CAMs in languages that they were not fluent in, thus resulting in screening of primarily English-speaking patients. Psychiatry was consulted at the discretion of the primary team physician to assist with diagnosis and management of delirium. As psychiatry consultation was left up to the discretion of the primary team physician, not all CAM-positive patients were evaluated. The psychiatrists conducted no routine evaluation on the CAM-negative patients unless requested by the primary team physician. The psychiatrist confirmed the delirium diagnosis with a clinical interview and assessment. The patients confirmed with delirium at any point during their hospitalization were prospectively added to a delirium registry. The patients assessed by the psychiatrist as not delirious were excluded from the registry. Only those patients added to the delirium registry during the study period were classified as delirious for this study. All other patients were included as controls. The presence of the nursing screening program using the CAM enriched the cohort, but a positive CAM was unnecessary nor was it sufficient for inclusion in the delirium group (Table 1).

To eliminate the influence of previous delirium episodes on readmission, the subjects were excluded if they reported a prior diagnosis of delirium in 2006 or later, which was the year the electronic medical record was initiated. This diagnosis was determined retrospectively using the following ICD-9 codes: 290.11, 290.3, 290.41, 292.0, 292.81, 292.89, 293.0, 293.0E, 293.0F, 293.1, 293.89, 294.10, 294.21, 304.00, 304.90, 305.50, 331.0, 437.0, 780.09 , V11.8, and V15.89. ${ }^{26}$ Subjects were also excluded if they were ever diagnosed with alcohol-related delirium, as defined 
by ICD-9 codes 291, 303.9, and 305. Subjects were excluded from the primary analysis if Kaiser Permanente membership lapsed to any degree within 30 days of discharge. Patients who died in the hospital were not excluded; however, the analyses of postdischarge outcomes were conducted on the subpopulation of study subjects who were discharged alive.

For subjects with multiple entries in the delirium registry, the earliest hospitalization during the study period in which a delirium diagnosis was recorded was selected. For eligible patients without a diagnosis of delirium, a single hospitalization was selected randomly from the individual patients during the time period. The analysis database included only one hospitalization for each subject. The flowchart of patient selection is outlined in the Figure.

\section{Patient Characteristics}

Patient demographics and clinical data were obtained from the electronic medical records. We used several scores to characterize illness severity, including the Charlson comorbidity index, ${ }^{27}$ Laboratory-Based Acute Physiology, version 2 (LAPS2) score ${ }^{28}$-an externally validated score for acute severity of illness-and disease categories as defined by the Healthcare Cost and Utilization Project (HCUP). ${ }^{29}$

\section{Outcomes}

The primary outcome was the rate of readmission to the hospital within 30 days of discharge from the hospitalization in which delirium was first diagnosed. Readmissions and ED visits to any Kaiser Permanente hospital and to hospitals outside of the Kaiser Permanente network with Kaiser Permanente insurance were captured. To avoid incorrectly coding patients transferred from the index hospital to another hospital as readmissions, we excluded readmissions that occurred on the day of discharge or the following calendar day. This action was expected to lower the absolute number of readmissions but restrict the analysis to true readmissions. The models of postdischarge outcomes are based on the subset of patients discharged alive. The secondary outcome measures included discharge from the index hospitalization to a skilled nursing facility or hospice rather than to home and emergency room visits within 30 days of discharge. We also quantified rates of mortality during hospitalization and at 30 days postdischarge.

\section{Statistical Analysis}

Comparisons between patients with delirium and those without were performed using Pearson's $X^{2}$ test for categorical variables and student $\mathrm{t}$-test for continuous variables. The estimated odds of our outcome measures for delirious and nondelirious subjects were calculated from multivariable logistic regression models, which controlled for predictors of delirium and additional information obtained during the hospitalization. For inpatient outcomes (in-hospital mortality and discharge to skilled nursing facility or hospice), we adjusted only for admission characteristics: age, race/ethnicity, admission to ICU, Charlson comorbidity index, HCUP category, and admission category. To limit the number of variables in our model, we
TABLE 1. Delirium Assessments Included in Exposed and Unexposed Groups

\begin{tabular}{lcc}
\hline Assessment & Delirium + (Exposed) & Delirium - (Unexposed) \\
\hline Nurse CAM Screen & CAM+ or CAM- & CAM+ or CAM- \\
\hline $\begin{array}{l}\text { Psychiatrist Delirium } \\
\text { Assessment }\end{array}$ & +DSM IV Delirium & -DSM IV Delirium or no psychiatry \\
assessment
\end{tabular}

Abbreviations: CAM, confusion assessment method; DSM IV, Diagnostic and Statistical Manual of Mental Disorders 4th edition.

consolidated the initial 30 HCUP categories (Appendix Table 1) by illness type into 13 categories (Appendix Table 2). For postdischarge outcomes, we adjusted for all the variables, including disposition (Table 2). The average estimated odds were calculated based on the observed marginal distribution of the control variables. The $P$ value indicates how likely the odds on each outcome for delirious subjects differed significantly from those for other subjects. All statistical analyses were performed using SAS 9.3 (SAS Institute Inc., Cary, North Carolina).

\section{RESULTS}

\section{Demographics and Clinical Characteristics}

A total of 718 patients with delirium and 7,927 patients without delirium were included in this study. The related demographic information is outlined in Table 2. On average, the patients with delirium were older $(83 \pm 8$ years versus $77 \pm 8$ years, $P<.0001)$ but no difference in gender distribution was observed between groups. A similar racial breakdown was noted between groups, with white patients accounting for $87 \%$ of both patients with delirium and those without. The majority of admissions were unplanned medical admissions. The delirium cohort included more emergent surgical admissions compared with patients who did not develop delirium. Patients who developed delirium exhibited higher levels of illness severity on admission, as measured by the Charlson and LAPS2 scores, and were more often admitted to the ICU. Significant differences were also observed between admission illness categories between patients with delirium and those without.

\section{Primary Outcome}

Delirium during admission was significantly associated with hospital readmission within 30 days of discharge (adjusted odds ratio $[\mathrm{aOR}]=2.60,95 \% \mathrm{Cl}: 1.96-3.44 ; P<.0001$; Table 3 ).

\section{Secondary Outcomes}

Delirium during admission was significantly $(P<.0001$; Table 3 ) associated with an ED visit within 30 days of discharge (OR: 2.18; 95\% Cl: 1.77-2.69) and discharge to a skilled nursing facility or hospice rather than home (OR: 2.52; 95\% Cl: 2.09-3.01). Delirium was not associated $(P>.1)$ with death during hospitalization nor death 30 days following discharge.

As the delirious patients were much more likely to be discharged to a skilled nursing facility than nondelirious patients, we tested whether discharge disposition influenced readmis- 
TABLE 2: Patient Demographics Featuring Raw Observational Cohort Data

\begin{tabular}{|c|c|c|c|}
\hline Variable & $\begin{array}{l}\text { Delirium } \\
(\mathrm{n}=718)\end{array}$ & $\begin{array}{c}\text { No Delirium } \\
(n=7,927)\end{array}$ & $P$ Value \\
\hline Age on Admission (years) & $83 \pm 8$ & $77 \pm 8$ & $<.0001$ \\
\hline Female & $409(57 \%)$ & $4,493(57 \%)$ & .9 \\
\hline Race & & & .6 \\
\hline White (NonHispanic) & $626(87 \%)$ & $6,900(87 \%)$ & \\
\hline Black & $7(1 \%)$ & $107(1 \%)$ & \\
\hline Hispanic & $24(3 \%)$ & $306(4 \%)$ & \\
\hline Asian & $18(3 \%)$ & $221(3 \%)$ & \\
\hline Not Elsewhere Classified & $43(6 \%)$ & $393(5 \%)$ & \\
\hline Admission Category & & & $<.0001$ \\
\hline Medical - Emergent & $483(67 \%)$ & $4,395(55 \%)$ & \\
\hline Medical - Planned & $59(8 \%)$ & $939(12 \%)$ & \\
\hline Surgical - Emergent & $115(16 \%)$ & $713(9 \%)$ & \\
\hline Surgical - Planned & $61(8 \%)$ & $1,880(24 \%)$ & \\
\hline Admission Location & & & $<.0001$ \\
\hline Intensive Care Unit & $64(9 \%)$ & $432(5 \%)$ & \\
\hline NonIntensive Care Unit & $654(91 \%)$ & $7,495(95 \%)$ & \\
\hline Charlson Score & $1.4 \pm 1.3$ & $1.0 \pm 1.5$ & $<.0001$ \\
\hline LAPS2 Score & $75.5 \pm 41.1$ & $47.8 \pm 36.6$ & $<.0001$ \\
\hline HCUP Category & & & $<.0001$ \\
\hline Acute Infection & $118(16 \%)$ & $588(7 \%)$ & \\
\hline Renal and Electrolyte Disorders & $11(2 \%)$ & $121(2 \%)$ & \\
\hline Neurologic and Psychiatric Disorders & $135(19 \%)$ & $806(10 \%)$ & \\
\hline Acute Cardiac Disease & $26(4 \%)$ & $357(5 \%)$ & \\
\hline Hip Fracture & $60(8 \%)$ & $184(2 \%)$ & \\
\hline Endocrine and Related Conditions & $17(2 \%)$ & $177(2 \%)$ & \\
\hline Gastrointestinal Disorders & $17(2 \%)$ & $403(3 \%)$ & \\
\hline Cancer & $10(1 \%)$ & $372(5 \%)$ & \\
\hline Other Infections & $23(3 \%)$ & $257(3 \%)$ & \\
\hline Low Acuity Conditions & $128(18 \%)$ & $2,316(29 \%)$ & \\
\hline Surgical Conditions & $73(10 \%)$ & $1,482(19 \%)$ & \\
\hline Trauma & $54(8 \%)$ & $402(5 \%)$ & \\
\hline Acute Pulmonary disease & $46(6 \%)$ & $462(6 \%)$ & \\
\hline Length of Hospital Stay (Days) & $7.4 \pm 9.3$ & $3.4 \pm 4.1$ & $<.0001$ \\
\hline Duration of time in ICU (Hours) & $25.8 \pm 114.1$ & $5.6 \pm 31.7$ & $<.0001$ \\
\hline Serum hemoglobin on discharge & $11.1 \pm 1.6$ & $11.5 \pm 1.7$ & $<.0001$ \\
\hline Serum sodium on discharge & $138.2 \pm 4.5$ & $137.2 \pm 3.9$ & $<.0001$ \\
\hline Disposition Location & & & $<.0001$ \\
\hline Home & $334(47 \%)$ & $6,373(80 \%)$ & \\
\hline Hospice & $58(8 \%)$ & $280(4 \%)$ & \\
\hline Skilled Nursing Facility & $284(40 \%)$ & $1,009(13 \%)$ & \\
\hline Death & $42(6 \%)$ & $265(3 \%)$ & \\
\hline \multicolumn{4}{|c|}{ Mean \pm standard deviation or total with percent from total subject group. } \\
\hline \multicolumn{4}{|c|}{$\begin{array}{l}\text { Abbreviations: HCUP, Healthcare Cost and Utilization Project ICU, intensive care unit; } \\
\text { LAPDS2, Laboratory Acute Physiology Score, version } 2 .\end{array}$} \\
\hline
\end{tabular}

sion rates and ED visits between delirious and nondelirious patients in an unadjusted univariate analysis. The association between delirium and readmission and ED utilization was present regardless of disposition. Among patients discharged to skilled nursing, readmission rates were $4.76 \%$ and $13.38 \%(P$ $<.001)$, and ED visit rates were $12.29 \%$ and $23.24 \%(P<.001)$ for nondelirious and delirious patients, respectively. Among patients discharged home, readmission rates were $4.96 \%$ and $14.37 \%(P<.001)$, and ED visit rates were $11.93 \%$ and $29.04 \%$ $(P<.001)$ for nondelirious and delirious patients, respectively.

\section{DISCUSSION}

In this study of patients in a community hospital in Northern California, we observed a significant association between inpatient delirium and risk of hospital readmission within 30 days of discharge. We also demonstrated increased skilled nursing facility placement and ED utilization after discharge among hospitalized patients with delirium compared with those without. Patients with delirium in this study were diagnosed by a psychiatrist—a gold standard ${ }^{30}$ —and the study was conducted in a health system database with near comprehensive ascertainment of readmissions. These results suggest that patients with delirium are particularly vulnerable in the posthospitalization period and are a key group to focusing on reducing readmission rates and postdischarge healthcare utilization.

Identifying the risk factors for hospital readmission is important for the benefit of both the patient and the hospital. In an analysis of Medicare claims data from 2003 to 2004, 19.6\% of beneficiaries were readmitted within 30 days of discharge. ${ }^{31}$ There is a national effort to reduce unplanned hospital readmissions for both patient safety as hospitals with high readmission rates face penalties from the Centers for Medicare and Medicaid Services. 22,23 Why delirium is associated with readmission remains unclear. Delirium may precipitate aspiration events, reduce oral intake which complicates medication administration and nutrition, or reduced mobility, leading to pulmonary emboli and skin breakdown, any of which could lead to readmission. ${ }^{32}$ Delirium may also accelerate the progression of cognitive decline and overall loss of functional independence. ${ }^{20}$ Delirious patients can be difficult to care for at home, and persistent delirium may lead to returns to the ED and readmission. Strategies to reduce readmissions associated with delirium may need to focus on both prevention of hospital-acquired delirium and targeted caregiver and patient support after discharge.

Hospital readmission and ED visits are not mutually exclusive experiences. In the United States, the majority of patients admitted to the hospital are admitted through the ED. ${ }^{33}$ Thus, most of the readmissions in this cohort were also likely counted as 30-day ED visits. However, as ED utilization occurs regardless of whether a patient is discharged or admitted from the ED, we reported all ED visits in this analysis, similar to other studies. ${ }^{34}$ More delirium patients returned to the ED 30 days postdischarge than were ultimately readmitted to the hospital, and delirious patients were more likely to visit the ED or be readmitted than nondelirious patients. These observations point toward the first 30 days after discharge as a crucial period for these patients. 
TABLE 3: Risk of Clinical outcomes in Patients with and without Delirium

\begin{tabular}{|c|c|c|c|c|}
\hline Clinical Outcome & $\begin{array}{l}\text { Delirium } \\
(95 \% \mathrm{Cl})\end{array}$ & $\begin{array}{l}\text { Controls } \\
(95 \% \mathrm{Cl})\end{array}$ & $\begin{array}{c}\text { Odds Ratio } \\
(95 \% \mathrm{Cl})\end{array}$ & $P$ Value \\
\hline Hospital Readmission 30 Days Postdischarge & $\begin{array}{c}5.7 \\
(3.5-9.1)\end{array}$ & $\begin{array}{c}2.2 \\
(1.4-3.3)\end{array}$ & $\begin{array}{c}2.60 \\
(1.96-3.44)\end{array}$ & $<.0001$ \\
\hline In-hospital Mortality & $\begin{array}{c}1.5 \\
(0.8-2.7)\end{array}$ & $\begin{array}{c}2.1 \\
(1.3-3.3)\end{array}$ & $\begin{array}{c}0.74 \\
(0.51-1.06)\end{array}$ & .1025 \\
\hline Emergency Department Visits 30 Days Postdischarge & $\begin{array}{c}20.6 \\
(15.1-28.2)\end{array}$ & $\begin{array}{c}9.5 \\
(7.3-12.3)\end{array}$ & $\begin{array}{c}2.18 \\
(1.77-2.69)\end{array}$ & $<.0001$ \\
\hline Mortality 30 Days Postdischarge & $\begin{array}{c}4.5 \\
(2.6-7.8)\end{array}$ & $\begin{array}{c}5.4 \\
(3.5-8.5)\end{array}$ & $\begin{array}{c}0.83 \\
(0.60-1.16)\end{array}$ & .2765 \\
\hline
\end{tabular}

Abbreviations: Cl, confidence interval; SNF, skilled nursing facility.

Our study features several strengths. To our knowledge, this study is one of the largest investigations of inpatients with delirium. One distinguishing feature was that all cases of delirium in this study were diagnosed by a psychiatrist, which is considered a gold standard. Many studies rely solely on brief nursing-administered surveys for delirium diagnosis. Using Kaiser Permanente data allowed for more complete follow-up of patients, including vital status. Kaiser Permanente is both a medical system and an insurer, resulting in acquisition of detailed health information from all hospitalizations where Kaiser Permanente insurance was used for each patient. Therefore, patients were only lost to follow-up following discharge in the event of a membership lapse; these patients were excluded from analysis. The obtained data are also more generalizable than those of other studies examining readmission rates in delirious patients as the hospital where these data were collected is a 116-bed general community medical and surgical hospital. Thus, the patients enrolled in this study covered multiple hospital services with a variety of admission diagnoses. This condition contrasts with much of the existing literature on inpatient delirium; these studies mostly center on specific medical conditions or surgeries and are often conducted at academic medical centers. At the same time, Kaiser Permanente is a unique health maintenance organization focused on preventive care, and readmission rates are possibly lower than elsewhere given the universal access to primary care for Kaiser Permanente members. Our results may not generalize to patients hospitalized in other health systems.

The diagnosis of delirium is a clinical diagnosis without biomarkers or radiographic markers and is also underdiagnosed and poorly coded. ${ }^{32}$ For these reasons, delirium can be challenging to study in large administrative databases or data derived from electronic medical records. We addressed this limitation by classifying the delirium patients only when they had been diagnosed by a staff psychiatrist. However, not all patients who screened positive with the CAM were evaluated by the staff psychiatrist during the study period. Thus, several CAM-positive patients who were not evaluated by psychiatry were included in the control population. This situation may cause bias toward identifi- cation of more severe cases of delirium. Although the physicians were encouraged to consult the psychiatry department for any patients who screened positive for delirium with the CAM, the psychiatrist may not have been involved if patients were managed without consultation. These patients may have exhibited less severe delirium or hypoactive delirium. In addition, the CAM fails to detect all delirious patients; interrater variability may occur with CAM administration, and non-English speaking patients are more likely to be excluded. ${ }^{35}$ These situations are another possible way for our control population to include some delirious patients and those patients with less severe or hypoactive subtypes. While this might bias toward the null hypothesis, it is also possible our results only indicate an association between more clinically apparent delirium and readmission. A major limitation of this study is that we were unable to quantify the number of cohort patients screened with the CAM or the results of screening, thus limiting our ability to quantify the impact of potential biases introduced by the screening program.

This study may have underestimated readmission rates. We defined readmissions as all hospitalizations at any Kaiser Permanente facility, or to an alternate facility where Kaiser Permanente insurance was used, within 30 days of discharge. We excluded the day of discharge or the following calendar day to avoid mischaracterizing transfers from the index hospital to another Kaiser Permanente facility as readmissions. This step was conducted to avoid biasing our comparison, as delirious patients are less frequently discharged home than nondelirious patients. Therefore, while the relative odds of readmission between delirious and nondelirious patients reported in this study should be generalizable to other community hospitals, the absolute readmission rates reported here may not be comparable to those reported in other studies.

Delirium may represent a marker of more severe illness or medical complications accrued during the hospitalization, which could lead to the associations observed in this study due to confounding. ${ }^{32}$ Patients with delirium are more likely to be admitted emergently, admitted to the ICU, and feature higher acuity conditions than patients without delirium. We attempted to mitigate this possibility by using a multivariable model 
to control for variables related to illness severity, including the Charlson comorbidity index, HCUP diagnostic categories, and ICU admission. Despite including HCUP diagnostic categories in our model, we were unable to capture the contribution of certain diseases with finer granularity, such as preexistent dementia, which may also affect clinical outcomes. ${ }^{36}$ Similarly, although we incorporated markers of illness severity into our model, we were unable to adjust for baseline functional status or frailty, which were not reliably recorded in the electronic medical record but are potential confounders when investigating clinical outcomes including hospital readmission.

We also lacked information regarding the duration of delirium in our cohort. Therefore, we were unable to test whether longer episodes of delirium were more predictive of readmission than shorter episodes.

\section{CONCLUSION}

In-hospital delirium is associated with several negative patient outcomes. Our study demonstrates that delirium predicts 30 day readmission and emergency department utilization after hospital discharge. Bearing in mind that a third of hospital-acquired delirium cases may be preventable, ${ }^{32}$ hospitals should prioritize interventions to reduce postdischarge healthcare utilization and complications in this particularly vulnerable group.

\section{Acknowledgments}

The authors would like to acknowledge Dr. Andrew L. Avins for his guidance with the initial development of this project and Julie Fourie for contributing data to the overall study.

Disclosures: Dr. Liu receives funding from NIH K23GM112018 and NIGMS R35128672. Dr. Josephson receives compensation as the JAMA Neurology Editor in Chief and Continuum Audio Associate Editor. The remaining authors have no conflicts of interest.

Funding: This study was funded by Kaiser Permanente Graduate Medical Education, who approved the design, conduct, and reporting of this study.

\section{References}

1. Bidwell J. Interventions for preventing delirium in hospitalized non-ICU patients: A Cochrane review summary. Int J Nurs Stud. 2017;70:142-143. doi 10.1016/j.ijnurstu.2016.11.010.

2. Ryan DJ, O'Regan NA, Caoimh RÓ, et al. Delirium in an adult acute hospital population: predictors, prevalence and detection. BMJ Open. 2013:3(1):e001772. doi: 10.1136/bmjopen-2012-001772.

3. McNicoll L, Pisani MA, Zhang Y, et al. Delirium in the intensive care unit: occurrence and clinical course in older patients. J Am Geriatr Soc. 2003;51(5):591 598. doi: 10.1034/j.1600-0579.2003.00201.x.

4. Inouye SK. Predisposing and precipitating factors for delirium in hospitalized older patients. Dement Geriatr Cogn Disord. 1999;10(5):393-400. doi: 10.1159/000017177.

5. Inouye SK, Zhang Y, Jones RN, et al. Risk factors for delirium at discharge: development and validation of a predictive model. Arch Intern Med. 2007;167(13):1406-1413. doi: 10.1001/archinte.167.13.1406.

6. LaHue SC, Liu VX. Loud and clear: sensory impairment, delirium, and functional recovery in critical illness. Am J Respir Crit Care Med. 2016;194(3):252253. doi: 10.1164/rccm.201602-0372ED.

7. Salluh JI, Soares M, Teles JM, et al. Delirium epidemiology in critical care (DECCA): an international study. Crit Care. 2010;14(6):R210. doi: 10.1186/cc9333.

8. Inouye SK, Charpentier PA. Precipitating factors for delirium in hospitalized elderly persons. Predictive model and interrelationship with baseline vulnerability. JAMA. 1996;275(11):852-857. doi: 10.1001/jama.275.11.852.

9. Ely EW, Shintani A, Truman B, et al. Delirium as a predictor of mortality in mechanically ventilated patients in the intensive care unit. JAMA 2004:291(14):1753-1762. doi: 10.1001/jama.291.14.1753.

10. Inouye SK, Westendorp RG, Saczynski JS. Delirium in elderly people. Lancet. 2014;383(9920):911-922. doi: 10.1016/S0140-6736(13)60688-1.
11. Brown EG, Douglas VC. Moving beyond metabolic encephalopathy: an update on delirium prevention, workup, and management. Semin Neurol. 2015;35(6):646-655. doi: 10.1055/s-0035-1564685.

12. Francis J, Martin D, Kapoor WN. A prospective study of delirium in hospitalized elderly. JAMA. 1990;263(8):1097-1101. doi: 10.1001/ jama.1990.03440080075027.

13. McCusker J, Cole MG, Dendukuri N, Belzile E. Does delirium increase hospital stay? J Am Geriatr Soc. 2003;51(11):1539-1546. doi: 10.1046/j.15325415.2003.51509.x

14. Salluh JI, Wang H, Schneider EB, et al. Outcome of delirium in critically ill patients: systematic review and meta-analysis. BMJ. 2015;350:h2538. doi: 10.1136/bmj.h2538.

15. Abelha FJ, Luís C, Veiga D, et al. Outcome and quality of life in patients with postoperative delirium during an ICU stay following major surgery. Crit Care. 2013;17(5):R257. doi: 10.1186/cc13084.

16. Siddiqi N, House AO, Holmes JD. Occurrence and outcome of delirium in medical in-patients: a systematic literature review. Age Ageing. 2006;35(4):350-364. doi: 10.1093/ageing/afl005.

17. Witlox J, Eurelings LS, de Jonghe JF, et al. Delirium in elderly patients and the risk of postdischarge mortality, institutionalization, and dementia: a meta-analysis. JAMA. 2010;304(4):443-451. doi: 10.1001/jama.2010.1013.

18. Inouye SK, Rushing JT, Foreman MD, Palmer RM, Pompei P. Does delirium contribute to poor hospital outcomes? A three-site epidemiologic study. J Gen Intern Med. 1998;13(4):234-242. doi: 10.1046/j.1525-1497.1998.00073.x.

19. Freter S, Koller K, Dunbar M, MacKnight C, Rockwood K. Translating delirium prevention strategies for elderly adults with hip fracture into routine clinical care: A pragmatic clinical trial. J Am Geriatr Soc. 2017;65(3):567-573. doi: 10.1111/jgs.14568.

20. Fong TG, Jones RN, Shi $P$, et al. Delirium accelerates cognitive decline in Alzheimer disease. Neurology. 2009;72(18):1570-1575. doi: 10.1212/ WNL.0b013e3181a4129a.

21. Girard TD, Jackson JC, Pandharipande PP, et al. Delirium as a predictor of long-term cognitive impairment in survivors of critical illness. Crit Care Med. 2010;38(7):1513-1520. doi: 10.1097/CCM.0b013e3181e47be1.

22. Berenson RA, Paulus RA, Kalman NS. Medicare's readmissions-reduction program-a positive alternative. N Engl J Med. 2012;366(15):1364-1366. doi: 10.1056/NEJMp1201268.

23. Desai NR, Ross JS, Kwon JY, et al. Association between hospital penalty status under the hospital readmission reduction program and readmission rates for target and nontarget conditions. JAMA. 2016;316(24):2647-2656. doi: 10.1001/jama.2016.18533.

24. Elsamadicy AA, Wang TY, Back AG, et al. Post-operative delirium is an independent predictor of 30-day hospital readmission after spine surgery in the elderly ( $\geq 65 y$ years old): a study of 453 consecutive elderly spine surgery patients. J Clin Neurosci. 2017;41:128-131. doi: 10.1016/j. jocn.2017.02.040.

25. Inouye SK, van Dyck CH, Alessi CA, et al. Clarifying confusion: the confusion assessment method. A new method for detection of delirium. Ann Intern Med. 1990;113(12):941-948. doi: 10.7326/0003-4819-113-12-941.

26. Inouye SK, Leo-Summers L, Zhang Y, et al. A chart-based method for identification of delirium: validation compared with interviewer ratings using the confusion assessment method. J Am Geriatr Soc. 2005;53(2):312-318. doi: 10.1111/j.1532-5415.2005.53120.x.

27. Charlson M, Szatrowski TP, Peterson J, Gold J. Validation of a combined comorbidity index. J Clin Epidemiol. 1994;47(11):1245-1251. doi: 10.1016/08954356(94)90129-5.

28. Escobar GJ, Gardner MN, Greene JD, Draper D, Kipnis P. Risk-adjusting hospital mortality using a comprehensive electronic record in an integrated health care delivery system. Med Care. 2013;51(5):446-453. doi: 10.1097/ MLR.0b013e3182881c8e

29. Steiner C, Elixhauser A, Schnaier J. The healthcare cost and utilization project: an overview. Eff Clin Pract. 2002;5(3):143-151.

30. Lawlor PG, Bush SH. Delirium diagnosis, screening and management. Curr Opin Support Palliat Care. 2014;8(3):286-295. doi: 10.1097/ SPC. 0000000000000062 .

31. Jencks SF, Williams MV, Coleman EA. Rehospitalizations among patients in the Medicare fee-for-service program. N Engl J Med. 2009;360(14):14181428. doi: 10.1056/NEJMsa0803563.

32. Fong TG, Tulebaev SR, Inouye SK. Delirium in elderly adults: diagnosis, prevention and treatment. Nat Rev Neurol. 2009;5(4):210-220. doi: 10.1038/nrneurol.2009.24.

33. Leyenaar JK, Lagu T, Lindenauer PK. Direct admission to the hospital: an alternative approach to hospitalization. J Hosp Med. 2016;11(4):303-305. doi: 10.1002/jhm.2512

34. Wang CL, Ding ST, Hsieh MJ, et al. Factors associated with emergency department visit within 30 days after discharge. BMC Health Serv Res. 2016;16:190. doi: 10.1186/s12913-016-1439-x.

35. Shi Q, Warren L, Saposnik G, Macdermid JC. Confusion assessment method: a systematic review and meta-analysis of diagnostic accuracy. Neuropsychiatr Dis Treat. 2013;9:1359-1370. doi: 10.2147/NDT.S49520.

36. Fick DM, Agostini JV, Inouye SK. Delirium superimposed on dementia: a systematic review. J Am Geriatr Soc. 2002;50(10):1723-1732. doi: 10.1046/j.15325415.2002.50468.x 